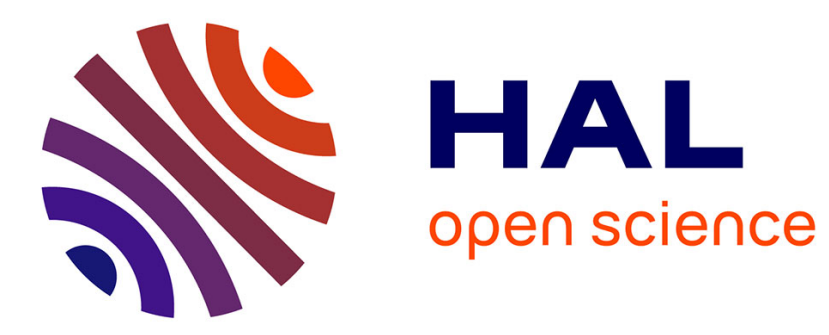

\title{
On the use of the Hs estimator for the experimental assessment of transmissibility matrices
}

Q. Leclere, Bert Roozen, Céline Sandier

\section{To cite this version:}

Q. Leclere, Bert Roozen, Céline Sandier. On the use of the Hs estimator for the experimental assessment of transmissibility matrices. Mechanical Systems and Signal Processing, 2014, 43 (1-2), pp.237-245. 10.1016/j.ymssp.2013.09.008 . hal-00911916

\section{HAL Id: hal-00911916 https://hal.science/hal-00911916}

Submitted on 10 Nov 2014

HAL is a multi-disciplinary open access archive for the deposit and dissemination of scientific research documents, whether they are published or not. The documents may come from teaching and research institutions in France or abroad, or from public or private research centers.
L'archive ouverte pluridisciplinaire HAL, est destinée au dépôt et à la diffusion de documents scientifiques de niveau recherche, publiés ou non, émanant des établissements d'enseignement et de recherche français ou étrangers, des laboratoires publics ou privés. 


\title{
On the use of the $H_{s}$ estimator for the experimental assessment of transmissibility matrices
}

\author{
Q. Leclère ${ }^{\mathrm{a}, *}$ N.B. Roozen ${ }^{\mathrm{b}, \mathrm{c}}$ C. Sandier ${ }^{\mathrm{a}}$ \\ ${ }^{a}$ Laboratoire Vibrations Acoustique, INSA-Lyon, 25 bis avenue Jean Capelle \\ F-69621 Villeurbanne Cedex, FRANCE \\ ${ }^{\mathrm{b}}$ Delft University of Technology, Faculty of Aerospace Engineering, Kluyverweg 1, \\ 2629 HS Delft, Netherlands \\ ${ }^{\mathrm{c}}$ Katholieke Universiteit Leuven, Department of Mechanical Engineering, Box \\ 2420, Celestijnenlaan 300 B, B-3001 Leuven, Belgium
}

\begin{abstract}
The experimental estimation of frequency response functions characterizing SISO linear systems is a well established topic. Several estimators are defined in the literature, each estimator being optimal depending upon the assumptions with respect to the balance of noise between the input and output of the system. $H_{1}$ and $H_{2}$ have to be used in case of presence of noise on output and input, respectively. The $H_{V}$ or $H_{s}$ estimator is chosen if input and output are assumed to have equivalent SNR. These estimators are also established for MIMO linear systems, with additional difficulties due to the necessity of inversing cross spectral matrices. A transmissibility function is generally defined as a linear relationship between two outputs of a linear system. For SIMO systems, transmissibility functions are uniquely defined. The $H_{s}$ estimator is thus advised if both outputs are of equivalent SNR. In the case of MIMO systems, transmissibility functions are no more defined by the system only, it also depends on the input quantities. It is however possible to define a transmissibility matrix between two sets of outputs that is, under some assumptions, uniquely defined. This approach is especially the base of Operational Transfer Path analysis, an engineering method benefiting of a strong research effort in the last few years. This paper deals with the use of the application of MIMO system estimators to the experimental assessment of transmissibility matrices. Transmissibility matrices are generally estimated using a $H_{1}$ like approach in the literature. The possibility of using $H_{2}$ and $H_{s}$ is presented in this work, from the theoretical point of view and with numerical and practical illustrations.
\end{abstract}

Key words: Transmissibility matrix , MIMO systems, linear system estimation, $H_{s}$ estimator 


\section{Introduction}

The transmissibility functions are generally defined as linear relationships between two reponses of a linear system. They have particular properties, in comparison with standard transfer functions representing classical excitationresponse relationships. The standard transfer function, for instance, is entirely defined by the studied system, while transmissibility between two responses depends also on the excitation configuration. Another important difference is that the standard transfer functions have peaks at the resonances of the system, while transmissibilities have peaks at frequencies corresponding to zeros of one of the considered response. Transmissibilities are thus more difficult to handle, but they are also easier to measure, because it is generally easier to mount a response sensor than an excitation sensor (which has to be inserted between the excitation device and the structure). That's why several transmissibility based methods have been developed in the literature, for instance in structural health monitoring [1], output only modal analysis [2,3], or Operational Transfer Path Analysis [4,5], in which the concept of transmissibility functions has to be extended to transmissibility matrices [6] between two sets of responses. The present work focusses on the experimental estimation of such transmissibility matrices.

Several estimators are known for the experimental assessment of transfer functions between one input and one output : $H_{1}$ has to be used when the noise is on the output and $\mathrm{H}_{2}$ when the noise is on the input [7]. Another estimator, $H_{s}$, has been proposed by Wicks and Vold [8], based on a total least squares approach, that consider noise on both the input and the output. This estimator has been extended to MIMO systems in [9] [10]. The $H_{s}$ approach seems particularly interesting for the estimation of transmissibility matrices, because the inputs and outputs of a transmissibility system are both responses, the SNR (Signal to Noise Ratio) has thus no reason to be higher on input or output responses.

The general principles of the transmissibility matrix approach is briefly treated in the first section of the paper. Then the concept of $H_{1}, H_{2}$, and $H_{s}$ estimates for transmissibility matrices is addressed from a theoretical point of view. The two last parts are dedicated to numerical and experimental illustrations, respectively.

N.B.: Throughout this paper, bold capitals are used for matrices (including

* Corresponding author. Fax: 33.4.72.43.87.12. E-mail address: quentin.leclere@insa-lyon.fr. 
vectors) and non-bold capitals for scalars. Note that all matrices are dependent upon frequency. For sake of brevity this dependency is not mentioned explicitly in the equations.

\section{Transmissibility matrices : definition}

Let us consider a linear dynamic system relating a set of $n$ excitation dofs $\mathbf{F}($ size $n \times 1)$ to two different sets of response dofs, named indicator dofs $\mathbf{Y}$ $(n \times 1)$ and output dofs $\mathbf{X}(m \times 1)$ :

$$
\left[\begin{array}{l}
\mathrm{X} \\
\mathrm{Y}
\end{array}\right]=\left[\begin{array}{l}
\mathbf{H} \\
\boldsymbol{\Phi}
\end{array}\right] \mathbf{F}
$$

where $\boldsymbol{\Phi}$ and $\mathbf{H}$ are transfer matrices relating excitation dofs to response dofs. A linear relationship can be then defined between $\mathbf{X}$ and $\mathbf{Y}$, under the condition of invertibility of $\boldsymbol{\Phi}$ :

$$
\mathbf{X}=\mathbf{H} \boldsymbol{\Phi}^{-1} \mathbf{Y}=\mathbf{T Y}
$$

where $\mathbf{T}=\mathbf{H} \boldsymbol{\Phi}^{-1}$ is the transmissibility matrix and where ${ }^{-1}$ denotes a matrix inverse. The existence of $\mathbf{T}$ thus depends on these two major conditions :

- the definition of a set of excitation dofs $\mathbf{F}$

- the invertibility of matrix $\boldsymbol{\Phi}$ relating $\mathbf{Y}$ to $\mathbf{F}$

\section{Transmissibility matrices : experimental estimation}

\subsection{Methodology}

The relation (2) between inputs and outputs can be written using cross spectral matrices

$$
\begin{aligned}
G_{x y} & =T G_{y y} \\
G_{x x} & =T G_{y x}
\end{aligned}
$$


where element $(p, q)$ of matrix $\mathbf{G}_{\mathbf{x y}}$ is the cross spectrum between the $p^{\text {th }}$ element of $\mathbf{X}$ and the $q^{\text {th }}$ element of $\mathbf{Y}$. However, the cross-spectral matrices obtained for a single operational condition are in general non invertible. Matrices $\mathbf{G}_{\mathbf{y y}}$ and $\mathbf{G}_{\mathbf{y x}}$ are indeed rarely of full rank and even less often well conditioned. A potential solution to this problem is to assess transmissibility matrices from non-stationary operating conditions, like run-up or down.

One approach is to gather several steady-state operating condition in one system:

$$
\mathbf{X}_{\mathbf{K}}=\left[\mathbf{X}_{1} \mathbf{X}_{2} \ldots \mathbf{X}_{k}\right] \quad \mathbf{Y}_{\mathbf{K}}=\left[\mathbf{Y}_{1} \mathbf{Y}_{2} \ldots \mathbf{Y}_{k}\right]
$$

where $\mathbf{X}_{i}$ and $\mathbf{Y}_{i}$ are response vectors obtained during one operating condition, using a phase reference sensor or more sophisticated techniques like Conditioned Spectral Analysis [7] or Virtual Source Analysis [11]. If more than one uncorrelated processes are identified (this is the case if the coherence function between channels is not close to unity), several response vectors can be extracted from each acquisition. Finally, cross spectral matrices can still be calculated from such results :

$$
\mathrm{G}_{\mathrm{xy}}=\mathrm{X}_{\mathrm{K}} \mathrm{Y}_{\mathrm{K}}^{\prime} \quad \mathrm{G}_{\mathrm{yy}}=\mathrm{Y}_{\mathrm{K}} \mathrm{Y}_{\mathrm{K}}^{\prime} \quad \mathrm{G}_{\mathrm{xx}}=\mathrm{X}_{\mathrm{K}} \mathrm{X}_{\mathrm{K}}{ }^{\prime}
$$

where' denotes the complex conjugate transpose. The experimental assessment of these matrices is not always easy, because the computation of $T$ requires inversions of these matrices, that have to be consequently of full rank. This condition is rarely fulfilled using only one operating condition of the studied system ; as was said earlier, it is often necessary to gather information from several operating conditions, using several steady state operating points or run-up-down acquisitions.

\section{2 $H_{1}$ and $H_{2}$ estimators}

For a scalar transfer function, the $H_{1}$ and $H_{2}$ estimators are given by

$$
\begin{aligned}
& H_{1}[T]=G_{x y} G_{y y}^{-1} \\
& H_{2}[T]=G_{x x} G_{y x}^{-1}
\end{aligned}
$$

The $H_{1}$ and $H_{2}$ estimates of the transmissibility matrix are, by analogy to equations (5) and (6) and by inversion of systems (3) and (4), defined by 


$$
\begin{aligned}
& H_{1}[\mathbf{T}]=\mathbf{G}_{\mathbf{x y}} \mathbf{G}_{\mathbf{y y}}{ }^{-1} \\
& H_{2}[\mathbf{T}]=\mathbf{G}_{\mathbf{x x}} \mathbf{G}_{\mathbf{y x}}{ }^{+}=\mathbf{G}_{\mathbf{x x}} \mathbf{G}_{\mathbf{y x}}\left(\mathbf{G}_{\mathbf{y x}} \mathbf{G}_{\mathbf{x y}}\right)^{-1}
\end{aligned}
$$

where ${ }^{+}$denotes the pseudo-inverse. It is worth nothing that the estimator $H_{2}[\mathbf{T}]$ requires $m \geq n$, which is a necessary condition for $\left(\mathbf{G}_{\mathbf{y x}} \mathbf{G}_{\mathbf{x y}}\right)$ to be of full rank.

\section{$3.3 H_{s}$ estimator}

For a scalar transfer function, the $H_{s}$ estimator is based on the eigenvalue decomposition of

$$
\left[\begin{array}{cc}
s^{2} G_{x x} & s G_{x y} \\
s G_{y x} & G_{y y}
\end{array}\right]=\left[\begin{array}{ll}
U_{x} & V_{x} \\
U_{y} & V_{y}
\end{array}\right]\left[\begin{array}{cc}
\lambda_{1} & 0 \\
0 & \lambda_{2}
\end{array}\right]\left[\begin{array}{ll}
U_{x} & V_{x} \\
U_{y} & V_{y}
\end{array}\right]^{\prime}
$$

where $s$ is a positive scaling factor used to balance the magnitude of $x$ and $y$. Assuming that the smallest eigenvalue is representing noise, $H_{s}$ is defined as the ratio between contributions of the largest eigenvalue $\left(\lambda_{1}\right)$ at $x$ and $y$ :

$$
H_{s}=\frac{U_{x}}{s U_{y}}
$$

which is explicitely given by the both following formulas

$$
\begin{aligned}
H_{s} & =\frac{s^{2} G_{x x}-G_{y y}+\sqrt{\left(s^{2} G_{x x}-G_{y y}\right)^{2}+4 s^{2}\left|G_{y x}\right|^{2}}}{2 s^{2} G_{y x}} \\
& =\frac{2 G_{x y}}{G_{y y}-s^{2} G_{x x}+\sqrt{\left(s^{2} G_{x x}-G_{y y}\right)^{2}+4 s^{2}\left|G_{y x}\right|^{2}}}
\end{aligned}
$$

It can be noted that eq. (10) is equivalent to $H_{2}$ when $s \rightarrow \infty$, and that eq. (11) is equal to $H_{1}$ when $s=0$.

The $H_{s}$ estimate of the transmissibility matrix is based on an analysis of the physical rank of the global cross spectral matrix. The system (1) can be formulated in terms of cross spectra :

$$
\mathbf{G}_{\mathbf{x y x y}}=\left[\begin{array}{ll}
\mathbf{G}_{\mathbf{x x}} & \mathbf{G}_{\mathbf{x y}} \\
\mathbf{G}_{\mathbf{y x}} & \mathbf{G}_{\mathbf{y y}}
\end{array}\right]=\left[\begin{array}{l}
\mathbf{H} \\
\Phi
\end{array}\right] \mathbf{S f f}\left[\begin{array}{l}
\mathbf{H} \\
\Phi
\end{array}\right]^{\prime}
$$


The columns of $\mathbf{G}_{\mathbf{x y x y}}$ are thus linear combinations of the columns of $\left[\mathbf{H}^{\prime} \Phi^{\prime}\right]^{\prime}$. The rank of $\mathbf{G}_{\mathbf{x y x y}}$ can not be greater than the number of input loads. The $H_{s}$ estimate of the transmissibility matrix is obtained from the following scaled eigenvalue decomposition

$$
\left[\begin{array}{cc}
\mathbf{s}_{\mathbf{x}} & 0 \\
0 & \mathbf{s}_{\mathbf{y}}
\end{array}\right]\left[\begin{array}{ll}
\mathbf{G}_{\mathbf{x x}} & \mathbf{G}_{\mathbf{x y}} \\
\mathbf{G}_{\mathbf{y x}} & \mathbf{G}_{\mathbf{y y}}
\end{array}\right]\left[\begin{array}{cc}
\mathbf{s}_{\mathbf{x}} & 0 \\
0 & \mathbf{s}_{\mathbf{y}}
\end{array}\right]=\left[\begin{array}{c}
\mathbf{U} \\
\mathbf{V}
\end{array}\right] \boldsymbol{\Lambda}\left[\begin{array}{l}
\mathbf{U} \\
\mathbf{V}
\end{array}\right]^{\prime}
$$

where $\Lambda$ is the diagonal matrix of eigenvalues, $\left[\mathbf{U}^{\prime} \mathbf{V}^{\prime}\right]^{\prime}$ the matrix of eigenvectors, and $\mathbf{s}_{\mathbf{x}}$ and $\mathbf{s}_{\mathbf{y}}$ diagonal scaling matrices. Considering that the number of input loads is equal to $n$ (as well as the number of indicators $\mathbf{Y}$ ), the rank of the (scaled) $\mathbf{G}_{\mathbf{x y x y}}$ matrix is lower or equal to $n$. The $m$ smallest singular values of $\boldsymbol{\Lambda}$ are thus considered as representing noise, and can be rejected:

$$
\left[\begin{array}{c}
\mathbf{U}_{\mathbf{n}} \\
\mathbf{V}_{\mathbf{n}}
\end{array}\right] \boldsymbol{\Lambda}_{\mathbf{n}}\left[\begin{array}{l}
\mathbf{U}_{\mathbf{n}} \\
\mathbf{V}_{\mathbf{n}}
\end{array}\right]^{\prime}=\left[\begin{array}{cc}
\mathbf{s}_{\mathbf{x}} & 0 \\
0 & \mathbf{s}_{\mathbf{y}}
\end{array}\right]\left[\begin{array}{c}
\mathbf{H} \\
\Phi
\end{array}\right] \mathbf{S}_{\mathbf{f f}}\left[\begin{array}{l}
\mathbf{H} \\
\Phi
\end{array}\right]^{\prime}\left[\begin{array}{cc}
\mathbf{s}_{\mathbf{x}} & 0 \\
0 & \mathbf{s}_{\mathbf{y}}
\end{array}\right]
$$

where $\boldsymbol{\Lambda}_{\mathbf{n}}$ the diagonal matrix of the $n$ largest eigenvalues and $\left[\mathbf{U}_{\mathbf{n}}{ }^{\prime} \mathbf{V}_{\mathbf{n}}{ }^{\prime}\right]^{\prime}$ are the $n$ corresponding eigenvectors. Let us write for convenience the eigenvalue decomposition of the cross spectral matrix of unknown forces :

$$
\mathrm{S}_{\mathrm{ff}}=\mathbf{P} \Sigma \mathbf{P}^{\prime}
$$

System (14) can be written as follows :

$$
\left\{\begin{array}{l}
\mathrm{U}_{\mathbf{n}} \Lambda_{\mathbf{n}} \mathrm{U}_{\mathbf{n}}^{\prime}=\mathbf{s}_{\mathbf{x}} \mathbf{H P} \Sigma \mathbf{P}^{\prime} \mathbf{H}^{\prime} \mathbf{s}_{\mathbf{x}} \\
\mathrm{V}_{\mathbf{n}} \Lambda_{\mathbf{n}} \mathrm{V}_{\mathbf{n}}^{\prime}=\mathbf{s}_{\mathbf{y}} \Phi \mathbf{P} \Sigma \mathbf{P}^{\prime} \Phi^{\prime} \mathbf{s}_{\mathbf{y}}
\end{array}\right.
$$

that can be written in a more simple way

$$
\left\{\begin{array}{l}
\mathbf{U}_{\mathbf{n}} \Lambda_{\mathbf{n}}{ }^{1 / 2}=\mathbf{s}_{\mathbf{x}} \mathbf{H P} \Sigma^{1 / 2} \\
\mathbf{V}_{\mathbf{n}} \Lambda_{\mathbf{n}}^{1 / 2}=\mathbf{s}_{\mathbf{y}} \boldsymbol{\Phi P} \Sigma^{1 / 2}
\end{array}\right.
$$

Then, $\boldsymbol{\Phi}^{-1}$ and $\mathbf{H}$ are expressed as follows

$$
\left\{\begin{aligned}
\mathbf{H} & =\mathbf{S}_{\mathbf{x}}{ }^{-1} \mathbf{U}_{\mathbf{n}} \boldsymbol{\Lambda}_{\mathbf{n}}{ }^{1 / 2} \boldsymbol{\Sigma}^{-1 / 2} \mathbf{P}^{\prime} \\
\boldsymbol{\Phi}^{-1} & =\mathbf{P} \boldsymbol{\Sigma}^{1 / 2} \boldsymbol{\Lambda}_{\mathbf{n}}{ }^{-1 / 2} \mathbf{V}_{\mathbf{n}}{ }^{-1} \mathbf{S}_{\mathbf{y}}
\end{aligned}\right.
$$


to finally obtain the expression of $H_{s}[\mathbf{T}]$

$$
H_{s}[\mathbf{T}]=\mathbf{H} \Phi^{-1}=\mathbf{s}_{\mathbf{x}}{ }^{-1} \mathbf{U}_{\mathbf{n}} \mathbf{V}_{\mathbf{n}}{ }^{-1} \mathbf{s}_{\mathbf{y}}
$$

It can be noted that if response vectors are extracted from several operating conditions to build $\mathbf{X}_{\mathbf{K}}$ and $\mathbf{Y}_{\mathbf{K}}$, the eigenvector decomposition of the whole matrix given by equation (13) can be replaced by the singular value decomposition :

$$
\left[\begin{array}{l}
\mathrm{s}_{\mathbf{x}} \mathbf{X}_{\mathbf{K}} \\
\mathbf{s}_{\mathbf{y}} \mathbf{Y}_{\mathbf{K}}
\end{array}\right]=\left[\begin{array}{l}
\mathrm{U} \\
\mathbf{V}
\end{array}\right] \text { A } \mathbf{W}^{\prime}=\left[\begin{array}{c}
\mathrm{U} \\
\mathbf{V}
\end{array}\right] \Psi
$$

where $\left[\mathbf{U}^{\prime} \mathbf{V}^{\prime}\right]\left[\begin{array}{l}\mathbf{U} \\ \mathbf{V}\end{array}\right]=\mathbf{W} \mathbf{W}^{\prime}=\mathbf{I}$, and where $\mathbf{A}$ is the diagonal matrix of singular values. The product $\mathbf{A} \mathbf{W}^{\prime}=\boldsymbol{\Psi}$ can be considered as a matrix of forces, and $\mathbf{U}$ and $\mathbf{V}$ as transfer matrices between $\boldsymbol{\Psi}$ and responses, respectively $\mathbf{X}_{\mathbf{K}}$ and $\mathbf{Y}_{\mathbf{K}}$ (cf. equation 1). Considering the number of forces exciting the structure equal to $\mathrm{n}$ as an a priori information, the $m$ smallest singular values can be zeroed. Finally, noting $\left[\mathbf{U}_{\mathbf{n}}{ }^{\prime} \mathbf{V}_{\mathbf{n}}{ }^{\prime}\right]^{\prime}$ the left singular vectors corresponding to the $n$ largest singular values, the expression of $H_{s}[\mathbf{T}]$ is the same as in equation (18). The computation of $\mathbf{U}_{\mathbf{n}}$ and $\mathbf{V}_{\mathbf{n}}$ leads indeed to equal results based on the eigen-decomposition of the whole cross spectral matrix (eq. 13) or from the singular value decomposition of the response vectors (eq. 20).

\subsection{Effects of scaling matrices on $H_{s}$}

The truncation of singular values can be seen as a way to denoise measurements, because smallest zeroed ones are considered as representing noise. The denoised $\mathbf{X}_{\mathbf{K}}$ and $\mathbf{Y}_{\mathbf{K}}$ matrices, noted $\tilde{\mathbf{Y}}_{\mathbf{K}}$ and $\tilde{\mathbf{Y}}_{\mathbf{K}}$, are then given by :

$$
\left[\begin{array}{c}
\tilde{X}_{\mathbf{K}} \\
\tilde{\mathbf{Y}}_{\mathbf{K}}
\end{array}\right]=\left[\begin{array}{l}
\mathbf{s}_{\mathbf{x}}{ }^{-1} \mathbf{U}_{\mathbf{n}} \\
\mathbf{s}_{\mathbf{y}}{ }^{-1} \mathbf{V}_{\mathbf{n}}
\end{array}\right] \quad \mathbf{A}_{\mathbf{n}} \mathbf{W}_{\mathbf{n}}{ }^{\prime}
$$

with $\mathbf{A}_{\mathbf{n}}$ the diagonal matrix of the $n$ largest singular values, and $\left[\mathbf{U}_{\mathbf{n}}{ }^{\prime} \mathbf{V}_{\mathbf{n}}{ }^{\prime}\right]^{\prime}$ and $\mathbf{W}_{\mathbf{n}}$ the corresponding left and right singular vectors, respectively.

Let us consider scaling matrices $\mathbf{s}_{\mathbf{y}}=s_{y} \mathbf{I}$ and $\mathbf{s}_{\mathbf{x}}=s_{x} \mathbf{I}$, with $\epsilon=s_{x} / s_{y}$ and $m=n$ (as many response as indicator sensors) for the sake of simplicity. Then it can be shown that: 


$$
\begin{array}{rll}
\lim _{\epsilon \rightarrow 0} \mathbf{A}_{\mathbf{n}}=\mathbf{A}_{\mathbf{Y}} & \lim _{\epsilon \rightarrow 0} \mathbf{W}_{\mathbf{n}}=\mathbf{W}_{\mathbf{Y}} & \lim _{\epsilon \rightarrow 0} \mathbf{V}_{\mathbf{n}}=s_{y} \mathbf{V}_{\mathbf{Y}} \\
\lim _{\epsilon \rightarrow \infty} \mathbf{A}_{\mathbf{n}}=\mathbf{A}_{\mathbf{X}} & \lim _{\epsilon \rightarrow \infty} \mathbf{W}_{\mathbf{n}}=\mathbf{W}_{\mathbf{X}} & \lim _{\epsilon \rightarrow \infty} \mathbf{U}_{\mathbf{n}}=s_{x} \mathbf{U}_{\mathbf{X}}
\end{array}
$$

where $\mathbf{A}_{\mathbf{Y}}$ and $\mathbf{W}_{\mathbf{Y}}$ are singular values and right singular vectors of $\mathbf{Y}_{\mathbf{K}}$, and $\mathbf{A}_{\mathbf{X}}$ and $\mathbf{W}_{\mathbf{X}}$ are singular values and right singular vectors of $\mathbf{X}_{\mathbf{K}}$, according to following SVDs

$$
\mathbf{X}_{\mathbf{K}}=\mathbf{U}_{\mathbf{X}} \mathbf{A}_{\mathbf{X}} \mathbf{W}_{\mathbf{X}}^{\prime} \quad \mathbf{Y}_{\mathbf{K}}=\mathbf{V}_{\mathbf{Y}} \mathbf{A}_{\mathbf{Y}} \mathbf{W}_{\mathbf{Y}}^{\prime}
$$

It means that the $n$ largest singular values are governed by only $\mathbf{Y}_{\mathbf{K}}$ when $\epsilon \rightarrow 0$ and by only $\mathbf{X}_{\mathbf{K}}$ when $\epsilon \rightarrow \infty$. In the first case, $\tilde{\mathbf{Y}}_{\mathbf{K}}$ is equal to $\mathbf{Y}_{\mathbf{K}}$ and rows of $\tilde{\mathbf{X}}_{\mathbf{K}}$ are projected on left singular vectors $\mathbf{W}_{\mathbf{Y}}$. In the second case, $\tilde{\mathbf{X}}_{\mathbf{K}}$ is equal to $\mathbf{X}_{\mathbf{K}}$ and lines of $\tilde{\mathbf{Y}}_{\mathbf{K}}$ are projected on left singular vectors $\mathbf{W}_{\mathbf{X}}$ :

$$
\lim _{\epsilon \rightarrow 0}\left[\begin{array}{c}
\tilde{\mathbf{X}}_{\mathbf{K}} \\
\tilde{\mathbf{Y}}_{\mathbf{K}}
\end{array}\right]=\left[\begin{array}{c}
\mathbf{X}_{\mathbf{K}} \mathbf{W}_{\mathbf{Y}} \mathbf{W}_{\mathbf{Y}}^{\prime} \\
\mathbf{Y}_{\mathbf{K}}
\end{array}\right] \quad \lim _{\epsilon \rightarrow \infty}\left[\begin{array}{c}
\tilde{\mathbf{X}}_{\mathbf{K}} \\
\tilde{\mathbf{Y}}_{\mathbf{K}}
\end{array}\right]=\left[\begin{array}{c}
\mathbf{X}_{\mathbf{K}} \\
\mathbf{Y}_{\mathbf{K}} \mathbf{W}_{\mathbf{X}} \mathbf{W}_{\mathbf{X}}{ }^{\prime}
\end{array}\right]
$$

which means that in the former case the noise is considered as contaminating $\mathbf{X}_{\mathbf{K}}$ only, and in the latter case $\mathbf{Y}_{\mathbf{K}}$ only. Limits of $\mathbf{U}_{\mathbf{n}}$ and $\mathbf{V}_{\mathbf{n}}$ when $\epsilon \rightarrow 0$ or $\infty$ are identified from the previous equations :

$$
\lim _{\epsilon \rightarrow 0} \mathbf{U}_{\mathbf{n}}=s_{x} \mathbf{X}_{\mathbf{K}} \mathbf{W}_{\mathbf{Y}} \mathbf{A}_{\mathbf{Y}}{ }^{-1} \quad \lim _{\epsilon \rightarrow \infty} \mathbf{V}_{\mathbf{n}}=s_{y} \mathbf{Y}_{\mathbf{K}} \mathbf{W}_{\mathbf{X}} \mathbf{A}_{\mathbf{X}}{ }^{-1}
$$

Finally, the limits of $H_{s}[\mathbf{T}]=\mathbf{s}_{\mathbf{x}}{ }^{-1} \mathbf{U}_{\mathbf{n}} \mathbf{V}_{\mathbf{n}}{ }^{-1} \mathbf{S}_{\mathbf{y}}$ when $\epsilon \rightarrow 0$ or $\infty$ are obtained :

$$
\begin{aligned}
\lim _{\epsilon \rightarrow 0} H_{s}[\mathbf{T}] & =\mathbf{X}_{\mathbf{K}} \mathbf{W}_{\mathbf{Y}} \mathbf{A}_{\mathbf{Y}}{ }^{-1} \mathbf{V}_{\mathbf{Y}}{ }^{\prime}=\mathbf{X}_{\mathbf{K}} \mathbf{Y}_{\mathbf{K}}{ }^{+}=H_{1}[\mathbf{T}] \\
\lim _{\epsilon \rightarrow \infty} H_{s}[\mathbf{T}] & =\mathbf{U}_{\mathbf{X}} \mathbf{A}_{\mathbf{X}}\left(\mathbf{Y}_{\mathbf{K}} \mathbf{W}_{\mathbf{X}}\right)^{-1}=\mathbf{X}_{\mathbf{K}} \mathbf{X}_{\mathbf{K}}\left(\mathbf{Y}_{\mathbf{K}} \mathbf{X}_{\mathbf{K}}\right)^{-1}=H_{2}[\mathbf{T}]
\end{aligned}
$$

These results show that the $H_{s}$ estimator for transmissibility matrices has a similar behavior with respect to $H_{1}$ and $H_{2}$ than for scalar transmissibilities. When the weight $\mathbf{s}_{\mathbf{y}}$ of indicator sensors increases, $H_{s}$ gets similar to $H_{1}$, and when the weight $\mathbf{s}_{\mathbf{x}}$ of response sensors increases, $H_{s}$ gets similar to $H_{2}$. In the former case the SNR will be a priori considered to be higher on indicator sensors $\mathbf{Y}_{\mathbf{K}}$ (i.e. more noise on $\mathbf{X}_{\mathbf{K}}$ ) and in the latter case on $\mathbf{X}_{\mathbf{K}}$ (i.e. more noise on $\left.\mathbf{Y}_{\mathbf{K}}\right)$.

The correct scaling of the system has to be done with respect to noise, but also with respect to different units or overall levels of each sensor. This is crucial if different type of sensors are used, for instance accelerometers and microphones. In such a case, a global scaling has to be applied so that overall scaled levels are almost equal (see $[12,13]$ for details). If this step is not correctly carried out, then the $H_{s}$ estimator will be arbitrarily closer to $H_{1}$ or $H_{2}$, not because 
of SNR balance assumptions but because of strong level differences due to the use of different units.

\subsection{An indicator for the validity of $H_{s}$}

The validity of equation (14) depends on the hypothesis that the $n$ largest eigenvalues are representing the signal, and the $m$ smallest ones the noise. This hypothesis can be verified by inspecting the ratio between the $n^{\text {th }}$ eigenvalue, representing the energy of the smallest incoherent process being part of signal, and the $(n+1)^{t h}$ eigenvalue representing the energy of largest noise component.

$$
R=\frac{\lambda_{n}}{\lambda_{n+1}}=\frac{a_{n}^{2}}{a_{n+1}^{2}}
$$

where $\lambda_{k}$ is the $k^{t h}$ largest eigenvalue of $\boldsymbol{\Lambda}$ in equation (13) and $a_{k}$ is the $k^{\text {th }}$ largest singular value of $\mathbf{A}$ in equation (20). Note that this check could also be performed to verify the rank of the cross-spectral matrix in case an $H_{1}$ or an $H_{2}$ matrix estimate is used. Indeed, whilst for $H_{1}$ and $H_{2}$ only $n$ singular values are extracted from $G_{y y}$ and $G_{y x}$, respectively, it is also for these estimators of prime importance that the physical rank of the cross-spectral matrix is equal to $n$.

\section{Numerical simulation}

A numerical simulation has been conducted to validate the proposed approach. An analytic model of a thin rectangular plate $\left(\left(L_{1} \times L_{2}\right)=(0.6 \times 0.5) m^{2}\right)$ has been used for simulations, with simply supported boundary conditions (physical parameters : aluminum, thickness $5 \mathrm{~mm}$, modal damping ratio $1 \%$ ). Three excitation points $\left(\left[x_{1}, x_{2}\right]=[0.05,0.01] ;[0.05,0.4] ;[0.45,0.25]\right)$ and six response points (same position as excitations for indicator responses $\mathbf{Y}$ and $\left[x_{1}, x_{2}\right]=[0.3,0.2] ;[0.5,0.42] ;[0.3,0.1]$ for output responses $\left.\mathbf{X}\right)$ have been considered. The response cross spectral matrix $\mathbf{G}_{\mathbf{x y x y}}$ has been computed using equation (12), using transfer matrices $\mathbf{H}$ and $\boldsymbol{\Phi}$ obtained with the analytical model and a cross spectral matrix $\mathbf{S}_{\mathrm{ff}}$ of uncorrelated unitary excitations. Measurement noise is added on the cross spectral matrix, using the approach described in appendix A. Gaussian incoherent noise is added on each indicator and output responses, with rms values adjusted to get a $30 \mathrm{~dB}$ SNR for all responses and with periodogram parameters $N=3000, M=100$. One element of the transmissibility matrix is drawn in Figure 1, directly computed using equation (2) from noise-free $\mathbf{H}$ and $\boldsymbol{\Phi}$ matrices, and estimated from noisy responses $G_{x y x y}$. All response channels are accelerations, and the global SNR is 
the same on all responses. Scaling factors are thus chosen equal to unity for this numerical illustration. It can be seen that $H_{s}$ behaves globally better than $H_{1}$ and $H_{2}$, particularly at low frequencies. In the low frequency range, the SNR is lower because the simulated response level is lower than in mid and high frequency. The global SNR is indeed fixed to $30 d B$, but the noise spectral density is constant (white noise) while the simulated accelerations are more energetic in high and mid frequency than in low frequency. The SNR spectrum is thus higher in mid and high frequency than in low frequency. Above $400 \mathrm{~Hz}$, the three estimations are in good agreement with the true transmissibility.

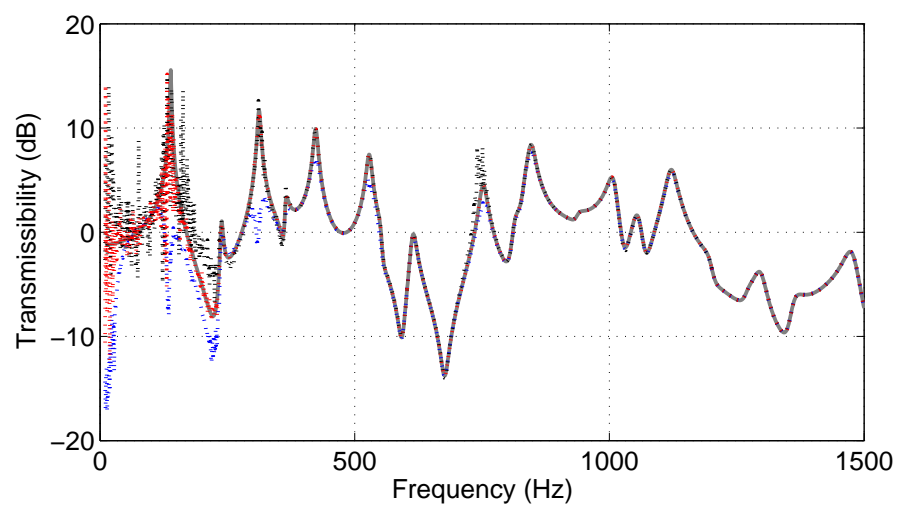

Fig. 1. \# $(3,1)$ element of the transmissibility matrix. reference $\mathrm{T}$ (Solid gray) $H_{1}$ (dotted blue), $H_{2}$ (dash-dotted black) and $H_{s}$ (dashed red) estimates

The estimation error is computed as a function of frequency for each estimator using

$$
E_{i}(f)=\frac{\left\|\mathbf{T}-H_{i}(\mathbf{T})\right\|_{F}}{\|\mathbf{T}\|_{F}}
$$

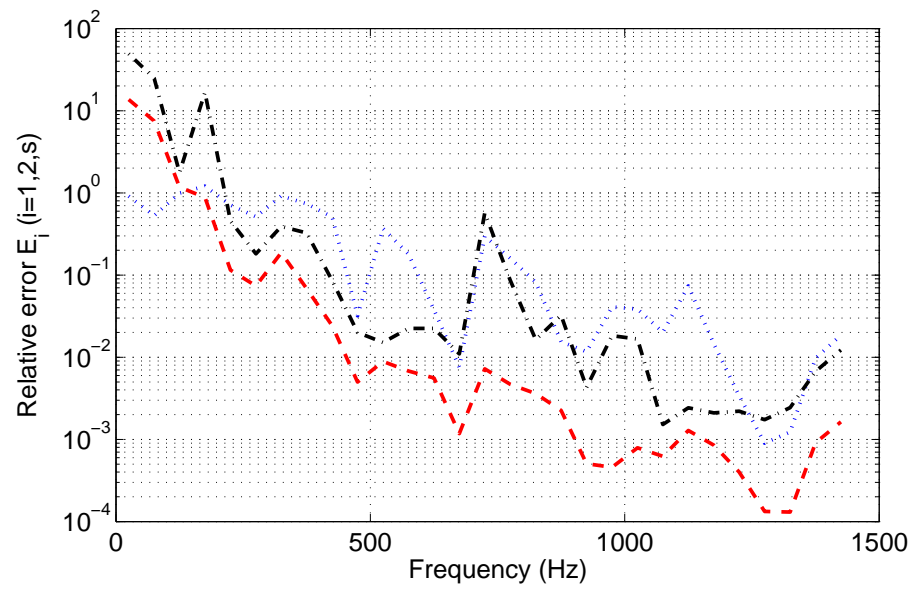

Fig. 2. $50 \mathrm{~Hz}$ frequency band integrated relative errors of $H_{1}$ (dotted blue), $H_{2}$ (dash-dotted black) and $H_{s}$ (dashed red) estimates. 
This error is drawn in Figure 2 for $i=1,2, s$. It is clear that the error $E_{s}$ is significantly lower than $E_{1}$ and $E_{2}$, except in low frequency (below $100 \mathrm{~Hz}$ ), where $E_{1}$ is the lowest one. This can be explained by a too low SNR in low frequency. If the SNR is too low, the eigenvalues representing noise in $G_{x y x y}$ can be higher than the eigenvalues representing the signal. In this case, keeping the $n$ largest eigenvalues leads to an erroneous estimation of $\mathbf{T}$. This can be illustrated by the value of the $H_{s}$ indicator $\mathrm{R}$ as introduced in section 3.5. This indicator $\mathrm{R}$ is equal to $0 \mathrm{~dB}$ below $200 \mathrm{~Hz}$, which means that the $n$ and $n+1^{\text {th }}$ eigenvalues are about the same level. It is thus hard to separate signal from noise. Above $200 \mathrm{~Hz}$, the ratio becomes significantly greater than 1 , resulting in a better estimation.

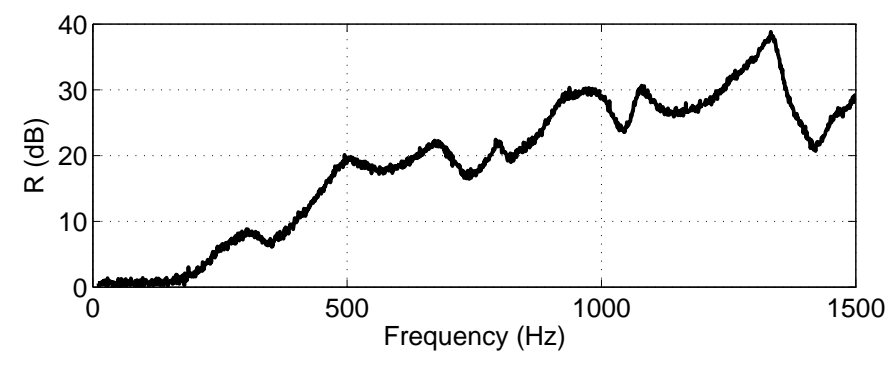

Fig. 3. $H_{s}$ indicator $\mathrm{R}$ given by equation (23)

In the current case, an equal amount of noise is used on all outputs to simulate measurement noise. For this reason $H_{s}$ with equal scaling factors gives the best estimate, as can be seen from Figure 2. In case different signal-to-noise ratios are present for the indicator responses $\mathbf{Y}$ and the output responses $\mathbf{X}$, a different scaling factor for the indicator responses $\mathbf{Y}$ and the output responses $\mathbf{X}$, respectively, would in that situation give the best $H_{s}$ estimate.

\section{Experimental illustration}

An experimental validation has been carried out to validate the proposed approach. The experiment took place in two rooms which are acoustically connected with each other by means of an aluminum plate with a thickness of $1 \mathrm{~mm}$ and a dimension of $60 \mathrm{x} 40 \mathrm{~cm}$. Twenty microphones -the set of output responses- were placed on the reception room side. The plate was excited from the emission room side by means of two shakers and one loudspeaker, constituting two structure borne paths and one airborne path. Two accelerometers were mounted on the plate, near shaker connection points, and one microphone was placed in the emission room : these three responses were chosen as indicators (the accelerometers for the two shakers and the microphone for the loudspeaker). In a first step, each source has been excited successively with white noise to measure directly transfer functions between inputs (signals sent to the shakers and loudspeaker) and responses, to build matrices $\mathbf{H}$ and $\mathbf{\Phi}$. 
For each excitation configuration with only one active source, the transfer functions have been estimated using a $H_{1}$ approach, which is well suited for transfer function measurements with a low noise on inputs. This assumption seems reasonable because input signals are directly measured (without acoustic or vibration transmission) and because they are white (the energy is distributed continuously on the whole frequency range). The transmissibility matrix assessed with $\mathbf{H}$ and $\boldsymbol{\Phi}$ (equation 2) is considered as the reference transmissibility matrix in the following.

In a second step, the three physical sources were driven by three uncorrelated generators simultaneously to measure the whole response cross-spectral matrix $\mathbf{G}_{\mathbf{x y x y}} \cdot H_{1}$ and $H_{2}$ estimators of the transmissibility matrix have been assessed, as well ad $H_{s}$. For the latter, a scaling has to be applied because different kinds of sensors are used (accelerometers and microphones). The scaling strategy was to normalize all measurement channel by its rms value, thus assuming that the SNR ratio is the same on all channels. One element of the transmissibility matrix is drawn in Figure 4. All estimators fit the reference transmissibility well on the whole frequency range, even if some errors are visible in low frequency.
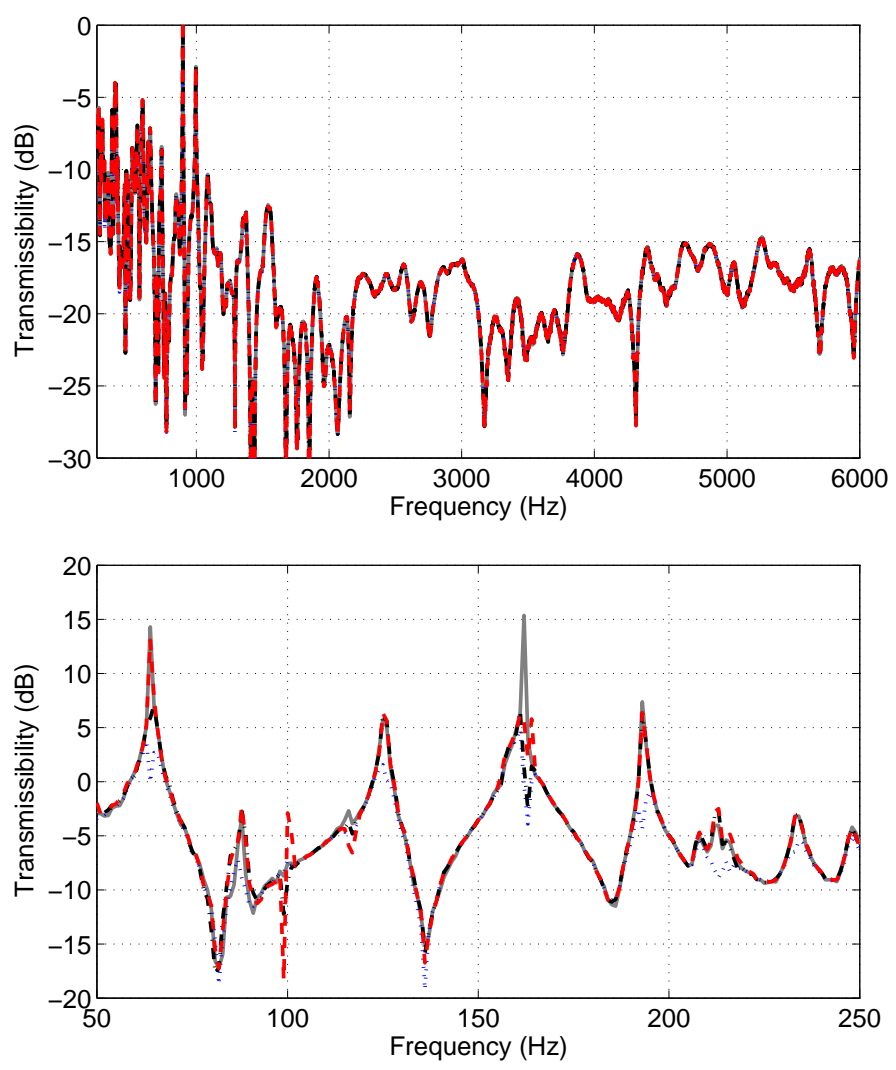

Fig. 4. \#(5,2) element of the transmissibility matrix, whole (top) and low (bottom) frequency ranges. Solid gray : $\mathrm{T}$ based on reference measurements, $H_{1}$ (dotted blue), $H_{2}$ (dash-dotted black) and $H_{s}$ (dashed red) estimates

The normalized errors, which are computed by means of equation 24, are 
drawn in Figure 5. It is clear that the estimation error is significantly lower with the $H_{s}$ estimator on the whole frequency range. In low frequency, $H_{s}$ and $H_{2}$ are similar, and globally better than $H_{1}$. In the high frequency range, $H_{s}$ is similar to $H_{1}$, even slightly better, and both are significantly better than $\mathrm{H}_{2}$.

It can be said globally that the $H_{s}$ estimator gives better results in this experiment than $H_{1}$ and $H_{2}$. The applied scaling strategy (normalization of the global levels) was however very basic, and could be improved and optimized to obtain even more satisfying results.

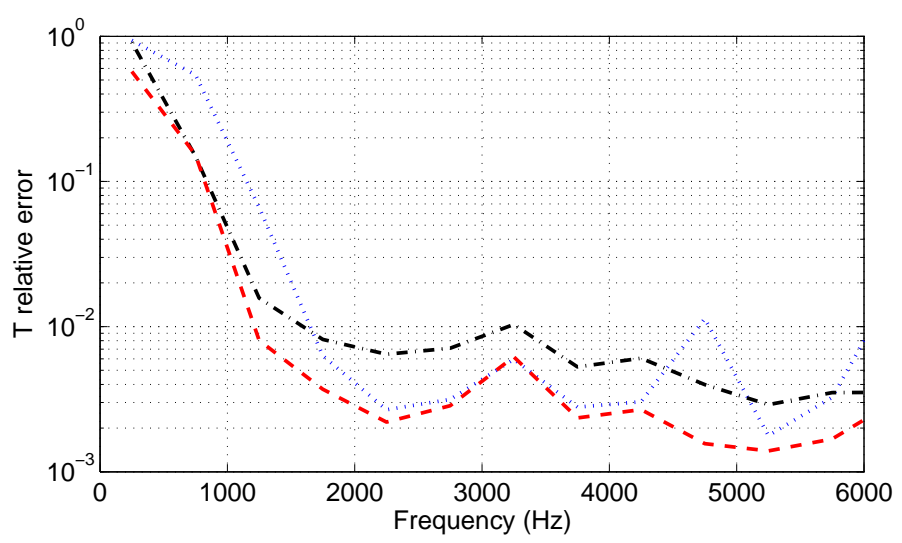

Fig. 5. $500 \mathrm{~Hz}$ frequency band integrated relative errors of $H_{1}$ (dotted blue), $H_{2}$ (dash-dotted black) and $H_{s}$ (dashed red) estimates.

The relative error of $H_{s}$ is drawn in Figure 6 (top) in narrow frequency bands in the low frequency range, together with the $R$ indicator defined in equation (23). It can be seen that the indicator is in good agreement with the estimation error : it can be said roughly that when the indicator is greater than $10 \mathrm{~dB}$, then the relative estimation error is below 10\%. On the other hand, when the indicator drops to almost 0dB, then the relative error increases and can exceed $100 \%$.

\section{Conclusion}

Transmissibility matrices are generally estimated using a $H_{1}$ like approach. The possibility to use $H_{2}$ and $H_{s}$ estimators for transmissibility matrices is presented. The $H_{s}$ approach is particularly interesting for the estimation of transmissibility matrices, because the inputs and outputs of a transmissibility system are both responses, the SNR (Signal to Noise Ratio) has thus no reason to be higher on input or output responses. The $H_{s}$ estimate of the transmissibility matrix is based on the eigenvalue decomposition of the global cross spectral matrix of input and output responses. After a rigorous derivation of the expressions to calculate $H_{s}$, it is theoretically shown that $H_{s}$ tends 

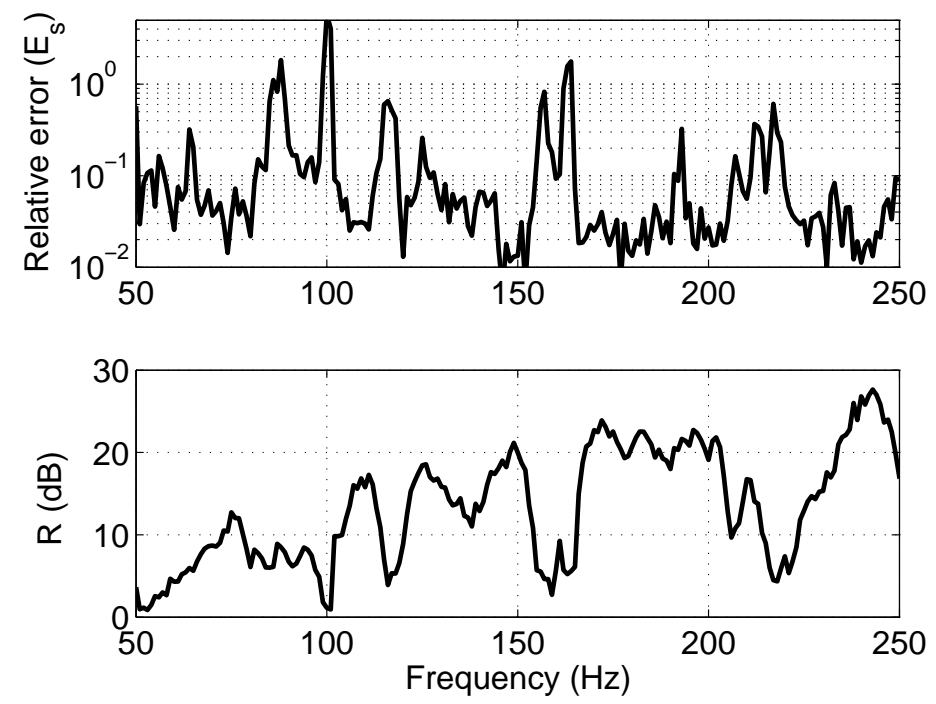

Fig. 6. Relative errors of $H_{s}[T]$ (top) and corresponding SNR indicator (bottom) in the low frequency range.

to $H_{1}$ and $H_{2}$, depending on the scaling ratios. It is worth nothing that the estimator $H_{2}$ only exists if the number of output dofs $m$ is equal or larger than the number of indicator dofs $n$. Both a numerical simulation and a physical experiment have been conducted to validate the proposed transmissibility estimates. In the numerical simulation a simply supported rectangular plate was considered. In the experiment a plate was excited by means of two shakers and one loudspeaker, constituting two structure borne paths and one airborne path. Both accelerometers and microphones were used, which required some scaling of the data in order to optimize the $H_{s}$ estimate. For both the numerical simulation and the physical experiment it was found that $H_{s}$ behaves globally better than $H_{1}$ and $H_{2}$. In addition, an indicator for the validity of $H_{s}$ is introduced, which checks that there is a clear separation between the $n$ largest eigenvalues (representing the signal), and the $m$ remaining eigenvalues (representing noise). By approximation, and at least for numerical and experimental illustrations presented in this work, it can be said that for values of the indicator larger than $10 \mathrm{~dB}$, the relative error in the transmissibility estimate is below $10 \%$. 


\section{Appendix A : simulation of cross-spectral matrices of uncorrelated finite gaussian signals estimated by the periodogram method}

The aim of this appendix is to explain how cross spectral matrices are randomly generated for measurement simulations. Studied signals are considered to be Gaussian :

$$
x[n]=\mathcal{N}(0 ; \bar{x}),
$$

where $\bar{x}$ stands for the rms value of the signal. The discrete Fourier Transform of $x$ is given by

$$
X_{k}=\frac{1}{N} \sum_{n=0}^{N-1} x[n] e^{-j 2 \pi k n / N}
$$

with the following expected value and variance

$$
\mathbf{E}\left(X_{k}\right)=0 \quad \mathbf{V}\left(X_{k}\right)=\bar{x}^{2} / N
$$

with $\mathbf{E}\left(X_{k}\right)=0$ and $\mathbf{V}\left(X_{k}\right)=\bar{x}^{2} / N . X_{k}$ follows a complex gaussian law for $k \neq\{0, N / 2\}$, the real and imaginary parts following real centered gaussian laws of variance $\bar{x}^{2} / 2 N$. For $k=\{0, N / 2\}, X_{k}$ follows a real centered gaussian law of variance $\bar{x}^{2} / N$, but this case will not be treated here for the sake of brevity. The double sided instantaneous autospectrum for $k \neq\{0, N / 2\}$ is equal to

$$
S_{x x_{k}}^{i}=2\left|X_{k}\right|^{2}=2 \mathcal{R}\left(X_{k}\right)^{2}+2 \mathcal{I}\left(X_{k}\right)^{2}=\frac{\bar{x}^{2}}{N} \chi_{2}^{2}
$$

with the following expected value and variance

$$
\mathbf{E}\left(S_{x x_{k}}^{i}\right)=\frac{2 \bar{x}^{2}}{N} \quad \mathbf{V}\left(S_{x x_{k}}^{i}\right)=\frac{4 \bar{x}^{4}}{N^{2}}
$$

The expected value and variance of the double sided instantaneous cross spectrum $S_{x y_{k}}^{i}=2 X_{k} Y_{k}^{*}$ of two independent signals $x$ and $y$ are

$$
\mathbf{E}\left(S_{x y_{k}}^{i}\right)=0 \quad \mathbf{V}\left(S_{x y_{k}}^{i}\right)=\frac{4 \bar{x}^{2} \bar{y}^{2}}{N^{2}}
$$

When applying the averaged periodogram method, auto and cross spectra are averaged over a number $M$ of time windows :

$$
S_{x x_{k}}=\left\langle\left|X_{k}\right|^{2}\right\rangle_{M} \quad S_{x y_{k}}=\left\langle X_{k} Y_{k}\right\rangle_{M}
$$

Assuming that $M$ is sufficiently high to apply the central limit theorem, then averaged auto and cross spectra are following gaussian distributions :

$$
\left.S_{x x_{k}}=\mathcal{N}\left(\frac{2 \bar{x}^{2}}{N} ; \frac{2 \bar{x}^{2}}{N \sqrt{M}}\right) \quad S_{x y_{k}}=\mathcal{N}\left(0 ; \frac{2 \bar{x} \bar{y}}{N \sqrt{M}}\right), \quad k \in\right] 0, N / 2[
$$


It is finally possible to simulate whole cross spectral matrices of uncorrelated signals using a gaussian random generator, from signals rms values, fixing values for $N$ (number of samples of a time window) and $M$ (number of time windows). 


\section{References}

[1] S. Chesné, A. Deraemaeker, and A. Preumont. On the transmissibility functions and their use for damage localization. In International Workshop on Structural Health Monitoring (IWSHM 09), Stanford, CA, USA, 2009.

[2] C. Devriendt and P. Guillaume. The use of transmissibility measurements in output-only modal analysis. Mechanical Systems and Signal Processing, 21(7):2689 - 2696, 2007.

[3] C. Devriendt, G. De Sitter, and P. Guillaume. An operational modal analysis approach based on parametrically identified multivariable transmissibilities. Mechanical Systems and Signal Processing, 24(5):1250 - 1259, 2010. Special Issue: Operational Modal Analysis.

[4] P. Gajdatsy, K. Janssens, Wim Desmet, and H. Van der Auweraer. Application of the transmissibility concept in transfer path analysis. Mechanical Systems and Signal Processing, 24(7):1963 - 1976, 2010. Special Issue: ISMA 2010.

[5] N. B. Roozen, Q. Leclere, and C. Sandier. Operational transfer path analysis applied to a small gearbox test set-up. In proceedings of Acoustics 2012, Nantes, France, 2012.

[6] A.M.R. Ribeiro, J.M.M. Silva, and N.M.M. Maia. On the generalisation of the transmissibility concept. Mechanical Systems and Signal Processing, 14(1):29 35,2000 .

[7] J.S. Bendat and A.G. Piersol. Engineering applications of correlation and spectral analysis. Wiley-Interscience, New York, 1980.

[8] A.L. Wicks and H. Vold. The hs frequency response function estimator. In Proc. 4th Int. Modal Analysis Conf., Los Angeles, CA, USA, 1986.

[9] P.R. White and W.B. Collis. Analysis of the tls frequency response function estimator. In Proceedings of the Ninth IEEE workshop on Statistical Signal Processing, Portland OR, USA, 1998.

[10] M.H. Tan and J.K. Hammond. A non-parametric approach for linear system identification using principal component analysis. Mechanical Systems and Signal Processing, 21(4):1576 - 1600, 2007.

[11] S.M. Price and R.J. Bernhard. Virtual coherence : A digital signal processing technique for incoherent source identification. In Proceedings of IMAC 4, Schenectady, NY, USA, 1986.

[12] Q. Leclere, C. Pezerat, B. Laulagnet, and L. Polac. Different least squares approaches to identify dynamic forces acting on an engine cylinder block. Acta Acustica, 90:285-292, 2004.

[13] Q. Leclere, C. Pezerat, B. Laulagnet, and L. Polac. Indirect measurement of main bearing loads in an operating diesel engine. Journal of Sound and Vibration, 286:341-361, 2005. 